\title{
Dynamic Responses in Polymer-Clay Gels
}

\author{
ELENA LOIZOU ${ }^{1}$, LIONEL PORCAR $^{2}$, PAUL BUTLER $^{2}$, MATTHEW MALWITZ $^{1}$, \\ GUDRUN SCHMIDT ${ }^{*}$
}

${ }^{1}$ Department of Chemistry, Louisiana State University, Baton Rouge, LA, 70803

${ }^{2}$ National Institute of Standards and Technology, Gaithersburg, MD 20899

\begin{abstract}
The shear orientation of three polymer-clay gels has been investigated by means of small angle neutron scattering (SANS). The gels have the same polymer and clay concentrations but different polymer molecular weight. The polymer is adsorbed to the clay platelets. While long polymer chains can interconnect several platelets shorter polymer chains cannot. Although the polymer concentration is above $\mathrm{c}^{*}$ the polymer chain length and cross linking between clay platelets strongly influence their shear orientation which leads to anisotropy in SANS. Our data suggest that the flow is strong enough to enhance and maintain a continuous increase in the shear orientation of the polymer clay gels only when the polymer chains are long enough to interconnect or strongly entangle between platelets.
\end{abstract}

\section{INTRODUCTION}

Polymer-clay gels, solutions and bulk nanocomposites are generating tremendous interest as novel materials exhibiting unique mechanical, electrical, optical, and thermal properties. The ability to tailor properties to the desired application by controlling nanoscopic structure can optimize the materials performance for a variety of applications. Nanocomposite applications range from pharmaceuticals, shampoos, optical switches to thin film barrier membranes and even ceramic precursor materials.

Several methods have been applied to examine the polymer-clay interactions and shear induced structural changes. Among them, microscopy and light, x-ray and neutron scattering are very powerful techniques for unveiling their structure and providing a measure of size, shape and interfacial polymer conformation. ${ }^{1-4}$ Solutions, gels as well as solution precursors experience considerable flow during processing and, sometimes, as part of their end use. Furthermore, many properties depend on the shear dependent structural changes such as the orientation of the clay platelets within the material, a property which can be greatly affected or controlled by shear flow. Thus it is imperative to understand the polymer clay interactions in solutions and their responses to shear.

The chemical simplicity of poly(ethylene-oxide) (PEO) makes it a useful model system for studying the charging effect induced by ion-dipole association in water. ${ }^{5-7}$ PEO strongly absorbs onto charged Laponite clay platelets. Low molar mass polymers thus inhibit aggregation of clay particles by classic steric hindrance, while higher molar mass polymers, particularly at higher concentrations of clay, bridge between particles and lead to the formation of large clusters ${ }^{8}$ or smart gels with novel properties. ${ }^{3,9-11}$ Recent small angle neutron scattering (SANS) studies describe the adsorption of PEO polymer chains onto Laponite clay platelets at low polymer and clay concentrations using contrast variation methods to separate the contributions from bulk and adsorbed polymer 
chains. ${ }^{12,13}$ Even more recently, SANS studies made with no excess polymer confirm these results but also find that the edges of the platelets have polymer density on them that is much thicker than on the face. ${ }^{2}$ Other research groups have used neutron diffraction to look at the local segmental, salt, and water structure around each clay platelet and to understand the mechanism of bridging flocculation. ${ }^{1,14}$

\section{EXPERIMENTAL}

In this study aqueous gels of the synthetic hectorite type clay, (Southern Clay) Laponite RD (LRD), and poly(ethylene-oxide) (PEO) were used. The clay particles are composed of platelets of high purity and relatively uniform size (25-30 nm in diameter and ca. $1 \mathrm{~nm}$ thick) and have a specific surface of $750 \mathrm{~m}^{2} / \mathrm{g}$. PEO with a $\mathrm{M}_{\mathrm{w}}=100 \times 10^{3}$, $600 \times 10^{3}$ and $10^{6} \mathrm{~g} / \mathrm{mol}$ was purchased from Polysciences Inc. For all samples in this study a $\mathrm{pH}$ value of ca. 10 and a $\mathrm{NaCl}$ concentration of $10^{-3} \mathrm{~mol} / \mathrm{L}$ were used. The results reported here were obtained from gels containing mass fractions of $3 \%$ LRD and $2 \%$ $\mathrm{PEO}$ at room temperature (LRD3-PEO2). At equilibrium, the clay surfaces are covered by adsorbed PEO segments with the diffuse polymer chains serving to "bridge" neighboring clay particles, leading to macroscopically homogeneous transparent hydrogels with near ideal clay exfoliation (no peaks in SANS at high q). ${ }^{3}$

SANS measurements were performed on the NG7 instrument at the Center for Neutron Research of the National Institute of Standards and Technology. The instrument is described well in literature. ${ }^{15}$ The shear cell is a Couette type geometry, which has been described previously. ${ }^{16}$ The cell has an inner diameter of $60 \mathrm{~mm}$ and a gap of $0.5 \mathrm{~mm}$ giving a total path length of $1 \mathrm{~mm}$ through the sample. In the standard configuration, referred to as the "radial beam" geometry, the incident beam is parallel to the shear gradient. The primary contrast in the SANS experiment is between $\mathrm{D}_{2} \mathrm{O}$ and the other solution components (clay, PEO), thus SANS experiments can detect the orientation of the clay platelets and polymer chains under shear. Small angle scattering investigations are excellent tools in providing quantitative information concerning structures on the 1 to ca. 1,000 nm length scales.

\section{RESULTS AND DISCUSSION}

The results obtained from three polymer clay gels are summarized in Figures 1 and 2. From present and previous results ${ }^{3}$ we know that at $3 \%$ LRD and $2 \%$ PEO solutions are well above the polymer overlap concentrations and the polymer chains are in a dynamic adsorption/desorption equilibrium with the clay particles to form a network. The distance between clay platelets in our gels (short relaxation of gel after cessation of shear) has been found before to be ca. $80 \mathrm{~nm}$ from a maximum or shoulder (structure factor, data not shown here). This value can be well correlated with the calculations of Callaghan and Ottewill ${ }^{17}$ and the experimental work done by Saunders et al. ${ }^{3,18}$ If however our sample is completely relaxed then no shoulder is visible (Figures $1 \& 2$ gels at rest) and the form factor dominates. Work done by Kroon et al who se approach treated clay particles as non interacting randomly distributed spheres determined a value of $33 \mathrm{~nm}$ for the distance between clay platelets. ${ }^{19}$ Depending on the model and the assumptions made for calculating interparticle distances different results are obtained. The origin of these discrepancies will be discussed in more detail in a future publication. 
If long enough, individual polymer chains may cross link between several platelets. While single "long" polymer chains such as PEO, $M_{w}=10^{6} \mathrm{~g} / \mathrm{mol}$, (hydrodynamic radius: $\mathrm{Rh}=100 \mathrm{~nm}$ measured from very dilute solutions) ${ }^{3}$ and $\mathrm{PEO}$, $\mathrm{M}_{\mathrm{w}}=600 \times 10^{3} \mathrm{~g} / \mathrm{mol},(\mathrm{Rh}=59 \mathrm{~nm}$,$) may be stretched and interconnect one or more clay$ platelets, individual short polymer chains such as $P E O, M_{w}=100 \times 10^{3} \mathrm{~g} / \mathrm{mol}$, $(\mathrm{Rh}=21 \mathrm{~nm})$ cannot. Although all samples have a polymer concentration above $\mathrm{c}^{*}$, the cross linking of individual polymer chains between clay platelets will strongly influence their shear orientation which leads to anisotropy in SANS. We have observed before, that a polymer clay solution with a polymer $\mathrm{Mw}=10^{6} \mathrm{~g} / \mathrm{mol}$ does strongly align while under similar conditions a comparable pure clay solution or a pure polymer solution does not shear orient. ${ }^{3}$ Our gels contain much more polymer than necessary to cover all clay platelets. Thus we expect the presence of excess polymer, which is not attached to the clay, to influence network structure on large length scales.

Figure 1a shows SANS data for a LRD3-PEO2-Mw100k gel in the q range of $(\mathrm{q}=4 \pi / \lambda \sin (\theta) / 2), 2.27 \times 10^{-3} \AA^{-1}<\mathrm{q}<0.24 \AA^{-1}$ and at different shear rates. SANS intensity versus $q$ was averaged radially. At rest an isotropic shoulder of scattering intensity was observed which becomes more diffuse at higher shear rates. Under shear the data clearly show lack of shear rate dependence at the high q regime which probes length scales up to slightly larger than an individual disc. No SANS anisotropy was detected. At low q we observe shear rate dependence suggesting change in large scale structures. The slope at high $\mathrm{q}$ of slightly more than the -2 expected for disc like structures ${ }^{14-17}$ is not surprising given that the free polymer coils also must contribute some to the scattering in this regime. Inserts of Figure 1a show isotropic 2D SANS patters as obtained from the sample at rest and at the highest measured shear rate. Both spectra have isotropic intensity distribution with the one at high shear rates exhibiting intensity increase around the beamstop, which corresponds to low q. Figure $1 \mathrm{~b}$ shows the low q scattering in more detail. While the structure on a nanometer length scale does not seem to change for the LRD3-PEO2-Mw100k gel (high q data), the "micrometer structure" such as aggregates or domain sizes does change (low q data). An apparent Rg can be obtained from a Guinier fit to the data at rest (no shear) and yields ca. $10 \mathrm{~nm}$ consistent with the $\mathrm{Rg}$ of $10.6 \mathrm{~nm}$ for $30 \mathrm{~nm}$ diameter discs. However, attempts to fit the full curves to a simple core+shell model similar to the one used by Nelson et $\mathrm{al}^{2}$ for dilute systems of PEO/laponite fails for the sample at rest as well as for the shear orientation. We conclude that the data summarized in Figure 1 show change in large scale structures with shear but on a nanometer length scale the data suggest that the flow is not strong enough to enhance and maintain a continuous increase in the orientation of the LRD3-PEO2-Mw100k system.

Figure 2 summarizes a) I versus q from a LRD3-PEO2-Mw600k gel and b) the anisotropy $\mid(\mathrm{Iz}-\mathrm{Iv})$ / (Iz+Iv)| from LRD3-PEO2-Mw100, 600, 1000k gels at different shear rates. Iz corresponds to the SANS intensity averaged horizontally and Iv is averaged vertically (insert Figure 2a). No SANS anisotropy was detected for the samples at rest. Similar to the 100k sample discussed above, a Guinier fit to the data observed at rest (Figure 2a) yields an $\mathrm{Rg}$ of ca $11 \mathrm{~nm}$. Given the same clay type and concentration for all samples this result is more or less expected. Under shear anisotropic scattering patterns developed. SANS intensity versus q was averaged in 10 degree sectors parallel and perpendicular to the flow direction. The inserts in Figure 2a show anisotropic 2D SANS patters with the horizontal and vertical sectors used for averaging $\mathrm{I}(\mathrm{q})$. Both $600 \mathrm{k}$ and 1000k samples show anisotropic scattering patterns when sheared, suggesting a shear 
rate of $90 \mathrm{~s}^{-1}$ is sufficient to induce orientation in both polymer-clay gels. The $1000 \mathrm{k}$ sample starts aligning below $10 \mathrm{~s}^{-1}$ while the 600k sample just starts aligning around a shear rate of $70 \mathrm{~s}^{-1}$ (Figure 2b) with intensity increase at low q (vertical average) and intensity decrease parallel to the flow (horizontal average, Figure 2a). SANS anisotropy suggests shear alignment of clay platelets and polymer on a nanometer length scale. At 90 $\mathrm{s}^{-1}$ the $1000 \mathrm{k}$ sample is strongly aligned (Figure $2 \mathrm{~b}$ ). The detailed shear orientation of the $1000 \mathrm{k}$ sample at even lower shear rates has been studied before. ${ }^{3,9}$
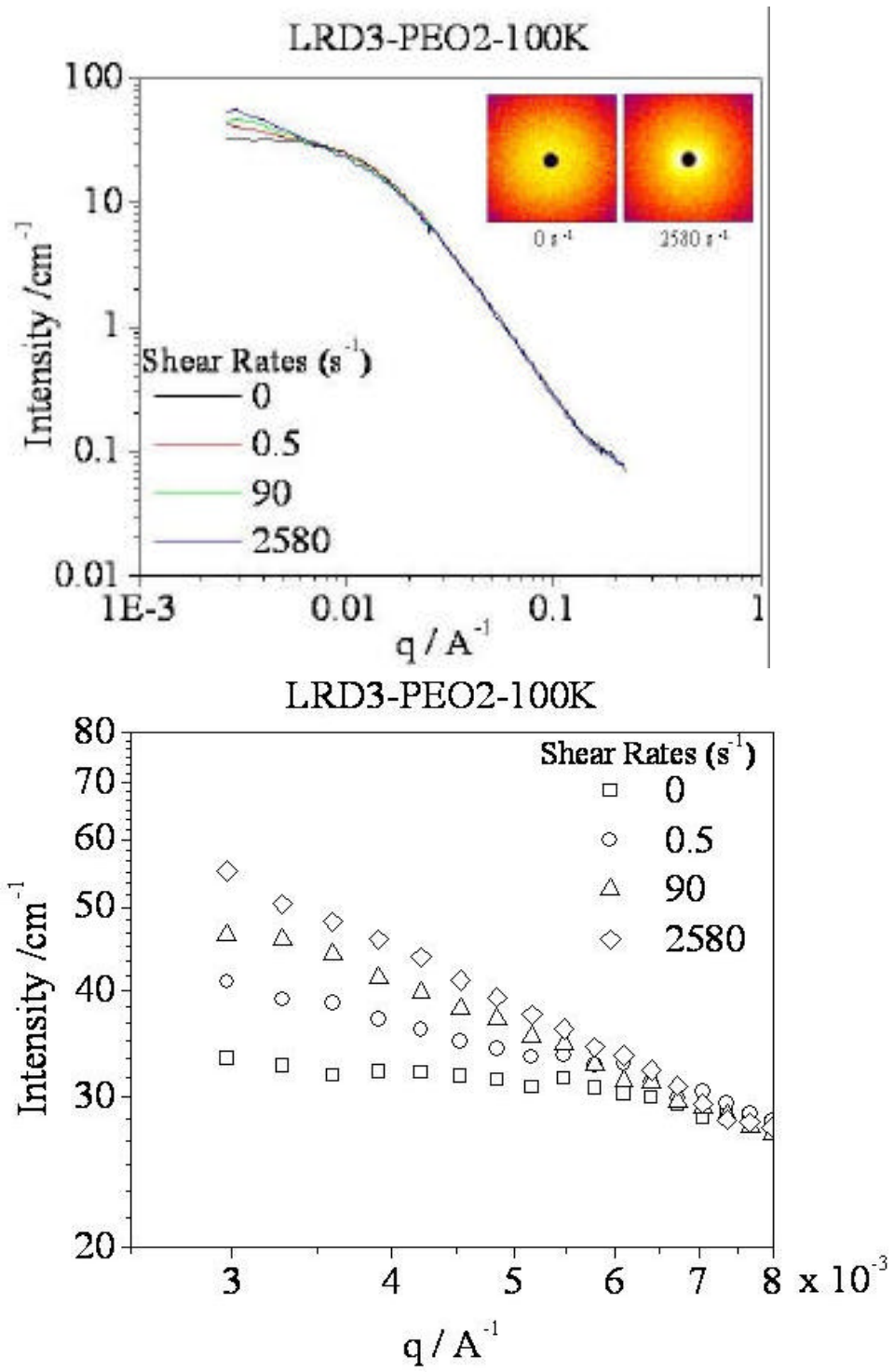

Figure 1. Top: a) Radial averaged SANS data for a LRD3-PEO2-Mw100k gel in the $q$ range of $(q=4 \pi / \lambda \sin (\theta) / 2), 2.27 \times 10^{-3} \AA^{-1}<q<0.24 \AA^{-1}$ and at different shear rates. Insert: isotropic 2D SANS patters as obtained from the sample at rest and the highest measured shear rate. Flow direction is horizontal. Bottom: b) Radial averaged low q SANS scattering at different shear rates. A relative error is smaller that the symbols. 

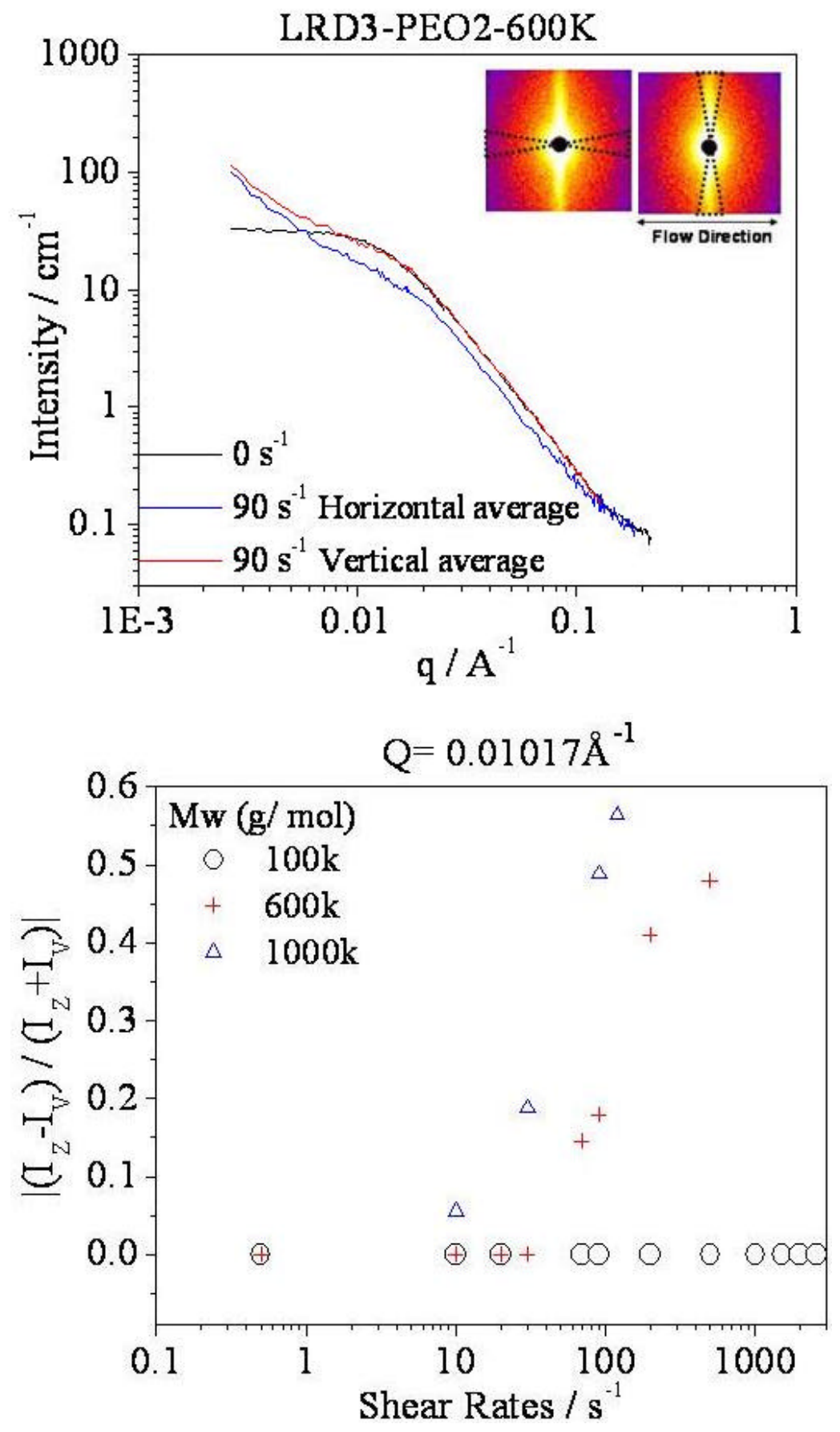

Figure 2. Top: a) Sector averaged SANS data for a LRD3-PEO2-Mw600k gel at rest (0s${ }^{1}$ ) and at a shear rate of $90 \mathrm{~s}^{-1}$. Insert: 2D SANS patters showing horizontal Iz and vertical Iv sectors used for averaging I(q). Flow direction is horizontal. Bottom: b) Anisotropy $(\mathrm{Iz}-\mathrm{Iv}) /(\mathrm{Iz}+\mathrm{Iv})$ for a $\mathrm{Q}$ of $0.01 \AA^{-1}$ as a function of shear rate for the 3 different PEO Mw's. Iz: horizontal averaged data, Iv: vertical averaged data

We conclude that the anisotropic SANS patters observed in the 600k and 1000k gels is due to the coupling between clay platelets and polymer, allowing a higher 
orientation with increasing polymer molecular weight. Individual long polymer chains such as 1000k PEO and 600k PEO may interconnect two or more clay platelets and are stretched under shear. The longer the polymer chains the more clay connections are possible. Individual "short" polymer chains such as 100k PEO cannot interconnect platelets (Figure 2b). Shear orientation of clay and stretching of polymer chains is not possible because the flow is not strong enough to enhance and maintain a continuous increase in the orientation of the 100k gel. Under similar conditions a comparable pure clay solution or a pure polymer solution does not shear orient. ${ }^{3}$

\section{ACKNOWLEDGMENTS}

This work was supported in part by an NSF-CAREER award, DMR 0348884. We also acknowledge the support of NIST and the NSF, through agreement no. DMR 9986442 in providing the neutron research facilities used in this work.

\section{REFERENCES}

(1) Swenson, J.; Smalley, M. V.; Hatharasinghe, H. L. M.; Fragneto, G. Langmuir 2001, 17, 3813-3818.

(2) Nelson, A.; Cosgrove, T. Langmuir 2004, 20, 2298-2304.

(3) Schmidt, G.; Nakatani, A. I.; Butler, P. D.; Han, C. C. Macromolecules 2002, 35, 4725-4732.

(4) Ho, D. L.; Glinka, C. J. Chemistry of Materials 2003, 15, 1309-1312.

(5) Hakem, I. F.; Lal, J. Appl Phys a-Mater 2002, 74, S531-S533.

(6) Hakem, I. F.; Lal, J. Europhys Lett 2003, 64, 204-210.

(7) Rixman, M. A.; Dean, D.; Ortiz, C. Langmuir 2003, 19, 9357-9372.

(8) Mongondry, P.; Nicolai, T.; Tassin, J. F. Journal of Colloid and Interface Science 2004, 275, 191-196.

(9) Schmidt, G.; Nakatani, A. I.; Butler, P. D.; Karim, A.; Han, C. C. Macromolecules 2000, 33, 7219-7222.

(10) Schmidt, G.; Nakatani, A. I.; Han, C. C. Rheol Acta 2002, 41, 45-54.

(11) Zebrowski, J.; Prasad, V.; Zhang, W.; Walker, L. M.; Weitz, D. A. Colloid Surface A 2003, 213, 189-197.

(12) Lal, J.; Auvray, L. Mol Cryst Liq Cryst 2001, 356, 503-515.

(13) Lal, J.; Auvray, L. J Appl Crystallogr 2000, 33, 673-676.

(14) Smalley, M. V.; Hatharasinghe, H. L. M.; Osborne, I.; Swenson, J.; King, S. M. Langmuir 2001, 17, 3800-3812.

(15) Glinka, C. J.; Barker, J. G.; Hammouda, B.; Krueger, S.; Moyer, J. J.; Orts, W. J. J Appl Crystallogr 1998, 31, 430-445.

(16) Straty, G. C.; Hanley, H. J. M.; Glinka, C. J. J Stat Phys 1991, 62, 1015-1023.

(17) Callaghan, I. C.; Ottewill, R. H. Faraday Discuss 1974, 57, 110.

(18) Saunders, J. M.; Goodwin, J. W.; Richardson, R. M.; Vincent, B. J Phys Chem B 1999, 103, 9211-9218.

(19) Kroon, M.; Vos, W. L.; Wegdam, G. H. Phys Rev E 1998, 57, 1962-1970. 